The following paper posted here is not the official IEEE published version. The final published version of this paper can be found in the Proceedings of the IEEE International Conference on

Acoustics, Speech and Signal Processing (2007 : Honolulu, Hawaii):pp.II-897-II-900

Copyright @ 2007 IEEE.

Personal use of this material is permitted. However, permission to reprint/republish this material for advertising or promotional purposes or for creating new collective works for resale or redistribution to servers or lists, or to reuse any copyrighted component of this work in other works must be obtained from the IEEE. 


\title{
SOURCE POWER ESTIMATION FOR ARRAY PROCESSING APPLICATIONS UNDER LOW SAMPLE SIZE CONSTRAINTS
}

\author{
Xavier Mestre ${ }^{1}$, Ben A. Johnson ${ }^{2}$, Yuri I. Abramovich ${ }^{3}$ \\ ${ }^{1}$ Centre Tecnològic de Telecomunicacions de Catalunya, Castelldefels, Barcelona (Spain) \\ xavier.mestre@cttc.cat \\ ${ }^{2}$ RLM Management, Pty. Ltd., Edinburgh, SA (Australia) \\ ben.a.johnson@ieee.org \\ ${ }^{3}$ Defence Science and Technology Organization, ISR Division, Edinburgh, SA (Australia) \\ yuri.abramovich@dsto.defence.gov.au
}

\begin{abstract}
This paper proposes a new power estimation technique for array processing applications in the low sample size regime. The technique is especially suitable for applications where the direction of arrival (DoA) detection is performed using subspace identification techniques, because the eigenvalues and eigenvectors of the sample covariance matrix are already computed for DoA estimation and are therefore available for power estimation as well. Compared to the traditional Maximum Likelihood (ML) power estimation technique, the proposed estimator has lower computational complexity (assuming that the eigendecomposition of the sample covariance matrix is already available) and it is more robust to the presence of outliers in the direction of arrival (DoA) detection process. This is because, contrary to the ML estimator, the proposed power estimator only depends on the signature of the source of interest.

Index Terms - Array signal processing, signal detection and estimation, G-estimation.
\end{abstract}

\section{INTRODUCTION}

We consider the problem of extracting the information of multiple uncorrelated sources impinging on an array of sensors. The number of signals present in the scenario is assumed to be known beforehand, so we are basically concerned with the joint estimation of the direction of arrival (DoA) and the power of the sources using low-complexity subspace-based techniques. We consider subspace-based techniques because they offer a good compromise between resolution and computational complexity, and are therefore very attractive from an implementation point of view.

Unfortunately, most of these array processing algorithms need to operate in situations where the number of available samples is not much higher (sometimes, even lower) than the number of sensors, and in that regime subspace-based algorithms are particularly vulnerable to the so-called performance breakdown effect $[1,2]$. The performance breakdown effect becomes especially relevant in superresolution direction-of-arrival (DoA) detection methods based on the MUSIC algorithm [3, 4]. For example, it is well known that MUSIC and its variants suffer from performance

This work was partially supported by the Catalan Government under grant SGR2005-00690 and the European Commission under project 6FP IST 26957. breakdown in scenarios where both classical order estimation techniques (such as those based on information theoretic criteria) and Maximum Likelihood (ML) estimators remain robust [5].

Quite recently, we presented in $[6,7]$ an alternative to traditional MUSIC that is able to alleviate, to some extent, this performance breakdown effect. The algorithm, which is referred to as GMUSIC, is derived by imposing consistency when both the number of samples and the number of elements of the array tend to infinity at the same rate. This guarantees a good behavior of the algorithm whenever these two quantities have the same order of magnitude, as it is the case in most practical situations. In this paper, we go one step further and propose a new source power estimation technique that is also consistent under the same asymptotic conditions. The algorithm is structured as a power estimation stage following DoA detection using GMUSIC and has several interesting properties, such as its low computational complexity (once GMUSIC has been implemented) and its robustness against outliers in the DoA detection process.

\section{SIGNAL MODEL AND SUBSPACE DOA DETECTION}

Let us consider a collection of $N$ complex valued array observations, $\mathbf{y}(n) \in \mathbb{C}^{M \times 1}, n=1 \ldots N$ obtained from an array of $M>1$ sensors. We consider the case where the array is receiving the signal from $K$ different uncorrelated sources, $K<M$, in white noise. In this case, the observations can be modeled to be independent, identically distributed random vectors with zero mean and covariance

$$
\mathbf{R}=\mathbf{S}(\Theta) \Phi_{S} \mathbf{S}(\Theta)^{H}+\sigma^{2} \mathbf{I}_{M}
$$

where $\mathbf{S}(\Theta)$ is an $M \times K$ matrix that contains the steering vectors corresponding to the $K$ different sources,

$$
\mathbf{S}(\Theta)=\left[\begin{array}{llll}
\mathbf{s}\left(\theta_{1}\right) & \mathbf{s}\left(\theta_{2}\right) & \cdots & \mathbf{s}\left(\theta_{K}\right)
\end{array}\right]
$$

$\Phi_{S}$ is a $K \times K$ diagonal matrix containing the power of each of the sources, $\Phi_{S}=\operatorname{diag}\left[P_{1}, \ldots, P_{K}\right]$, and $\sigma^{2}$ is the noise power.

Now, let $\left\{\mathbf{e}_{i}, i=1 \ldots M\right\}$ and $\lambda_{1} \leq \lambda_{2} \leq \ldots \leq \lambda_{M}$ denote the eigenvectors and associated eigenvalues of the true covariance matrix. According to the structure of the covariance matrix in (1), the lowest eigenvector has multiplicity $M-K$ and is exactly equal to the noise power, namely $\lambda_{1}=\ldots=\lambda_{M-K}=\sigma^{2}$. Hence, we 
can express the true covariance matrix as

$$
\mathbf{R}=\mathbf{E}_{S} \boldsymbol{\Lambda}_{S} \mathbf{E}_{S}^{H}+\sigma^{2} \mathbf{E}_{N} \mathbf{E}_{N}^{H}
$$

where $\boldsymbol{\Lambda}_{S}$ is a $K \times K$ diagonal matrix containing the largest eigenvalues of $\mathbf{R}, \mathbf{E}_{S}$ is an $M \times K$ matrix that contains the (signal) eigenvectors corresponding to the $K$ largest (signal) eigenvalues, and $\mathbf{E}_{N}$ is an $M \times M-K$ matrix that contains the (noise) eigenvectors associated with the smallest (noise) eigenvalue $\sigma^{2}$.

Subspace identification algorithms are based on the property that any vector lying on the signal subspace is orthogonal to the columns of $\mathbf{E}_{N}$. In this sense, the main idea behind the MUSIC approach consists in finding $\left\{\theta_{k}, k=1 \ldots K\right\}$ as the values of $\theta$ such that the column vector $\mathbf{s}(\theta)$ lies on the signal subspace of $\mathbf{R}$ or, equivalently, the values of $\theta$ such that

$$
\mathbf{s}^{H}(\theta) \mathbf{E}_{N} \mathbf{E}_{N}^{H} \mathbf{s}(\theta)=0
$$

In practice, the eigenvectors $\left\{\mathbf{e}_{i}\right\}$ are not known, and must be estimated from the received data. The MUSIC algorithm follows from the approach described above, replacing the unknown matrix of the true eigenvectors $\mathbf{E}_{N}$ with its sample estimates [3, 4]. Hence, if we denote by $\left\{\hat{\mathbf{e}}_{i}, i=1 \ldots M\right\}$ and $\left\{\hat{\lambda}_{1} \leq \hat{\lambda}_{2} \leq \ldots \leq \hat{\lambda}_{M}\right\}$ the eigenvectors and associated eigenvalues of the sample covariance matrix $\hat{\mathbf{R}}=\frac{1}{N} \sum_{n=1}^{N} \mathbf{y}(n) \mathbf{y}^{H}(n)$, the MUSIC estimation of the quadratic cost function in (3) turns out to be

$$
\eta_{\text {MUSIC }}(\theta)=\mathbf{s}^{H}(\theta)\left(\sum_{m=1}^{M-K} \hat{\mathbf{e}}_{m} \hat{\mathbf{e}}_{m}^{H}\right) \mathbf{s}(\theta) .
$$

Of course, the quadratic form $\eta_{\text {MUSIC }}(\theta)$ does not need to be zero anywhere, so that the estimated DoAs $\left\{\theta_{k}, k=1 \ldots K\right\}$ are selected as the $K$ deepest local minima of $\eta_{\text {MUSIC }}(\theta)$. When the number of samples increases without bound $(N \rightarrow \infty)$ and the observation dimension is taken to be a fixed quantity $(M<\infty)$, $\eta_{\text {MUSIC }}(\theta)$ tends almost surely to the deterministic original objective function $\mathbf{s}^{H}(\theta) \mathbf{E}_{N} \mathbf{E}_{N}^{H} \mathbf{s}(\theta)$. In other words, $\eta_{\text {MUSIC }}(\theta)$ is a (strongly) $N$-consistent estimator of (3). In practice, however, the number of samples $(N)$ is finite and has a similar order of magnitude as the observation dimension $(M)$. Hence, it makes more sense to use an estimator of this quadratic cost function that is consistent, not only when the number of samples increases without bound $N \rightarrow \infty$, but also when the observation dimension $M$ tends to infinity at the same rate, namely when $M, N \rightarrow \infty$ for $M / N \rightarrow c, 0<c<\infty$. These estimators, usually referred to as G-estimators [8] or $M, N$-consistent estimators, have a very good behavior in practical sample-size limited situations, where $M$ and $N$ have the same order of magnitude. It was shown in $[6,7]$ that, under some statistical assumptions and for sufficiently low $c$, this estimator takes the form

$$
\eta_{\text {GMUSIC }}(\theta)=\mathbf{s}^{H}(\theta)\left(\sum_{m=1}^{M} \phi(m) \hat{\mathbf{e}}_{m} \hat{\mathbf{e}}_{m}^{H}\right) \mathbf{s}(\theta)
$$

where

$\phi(m)=\left\{\begin{array}{cc}1+\sum_{k=M-K+1}^{M}\left(\frac{\hat{\lambda}_{k}}{\hat{\lambda}_{m}-\hat{\lambda}_{k}}-\frac{\hat{\mu}_{k}}{\hat{\lambda}_{m}-\hat{\mu}_{k}}\right) & m \leq M-K \\ -\sum_{k=1}^{M-K}\left(\frac{\hat{\lambda}_{k}}{\hat{\lambda}_{m}-\hat{\lambda}_{k}}-\frac{\hat{\mu}_{k}}{\hat{\lambda}_{m}-\hat{\mu}_{k}}\right) & m>M-K\end{array}\right.$

and where $\hat{\mu}_{1} \leq \hat{\mu}_{2} \leq \ldots \leq \hat{\mu}_{M}$ are the real-valued solutions to the following equation in $\hat{\mu}$

$$
\frac{1}{M} \sum_{k=1}^{M} \frac{\hat{\lambda}_{k}}{\hat{\lambda}_{k}-\hat{\mu}}=\frac{1}{c}
$$

repeated according to their multiplicity. When $c>1$, we use the convention $\hat{\mu}_{1}=\ldots=\hat{\mu}_{[M-N]^{+}+1}=0$, whereas $\hat{\mu}_{[M-N]++2}, \ldots, \hat{\mu}_{M}$ contain the positive solutions to the above equations. The GMUSIC algorithm, which selects the estimated DoAs as the $K$ deepest local minima of $\eta_{\text {GMUSIC }}(\theta)$, has been shown to outperform the traditional MUSIC algorithm in the low sample size regime.

\section{TRADITIONAL POWER ESTIMATION}

Once the DoAs of the $K$ different sources impinging on the array have been estimated, it remains the problem of determining their corresponding power $P_{1}, \ldots, P_{K}$. A very common approach is to use the Gaussian Maximum Likelihood estimator obtained without imposing zero correlation between the different sources, namely (see, for instance, [9])

$$
\hat{P}_{k}^{\mathrm{GML}}=\left\{\mathbf{A}^{H}(\hat{\Theta})\left(\hat{\mathbf{R}}-\hat{\sigma}_{\mathrm{GML}}^{2} \mathbf{I}_{M}\right) \mathbf{A}(\hat{\Theta})\right\}_{k k}
$$

where $\mathbf{A}(\hat{\Theta})=\mathbf{S}(\hat{\Theta})\left(\mathbf{S}^{H}(\hat{\Theta}) \mathbf{S}(\hat{\Theta})\right)^{-1}, \mathbf{S}(\hat{\Theta})$ is as in (2) but replacing the true DoAs with their corresponding estimates, $\{\cdot\}_{k k}$ denotes the $k$ th element of the diagonal and

$$
\begin{gathered}
\hat{\sigma}_{\mathrm{GML}}^{2}=\frac{1}{M-K} \operatorname{tr}\left[\hat{\mathbf{R}} \mathbf{P}_{\mathbf{S}}^{\perp}\right] \\
\mathbf{P}_{\mathbf{S}}^{\perp}=\mathbf{I}_{M}-\mathbf{S}(\hat{\Theta})\left(\mathbf{S}^{H}(\hat{\Theta}) \mathbf{S}(\hat{\Theta})\right)^{-1} \mathbf{S}^{H}(\hat{\Theta}) .
\end{gathered}
$$

Now, this estimator has two main drawbacks. First, the computational complexity associated with the computation of $\hat{P}_{k}^{\mathrm{GML}}$ is not low, especially when the number of sources $K$ is high. Second and more important, the power estimate associated with a particular source depends on the DoA of all the sources in the scenario. Therefore, the presence of one outlier in the DoA estimation may adversely effect even the power estimation of the sources whose DoA has been correctly detected.

There exist in the literature other estimators of the power of the sources that only depend on the signature of the source of interest. Here, we will consider the estimator proposed in [10], which can be particularly simple in computational complexity terms if the DoA detection has been performed using subspace approaches. The following identity was proven in [10]

$$
P_{k}=\frac{1}{\mathbf{s}^{H}\left(\theta_{k}\right)\left(\mathbf{R}-\sigma^{2} \mathbf{I}_{M}\right)^{\#} \mathbf{s}\left(\theta_{k}\right)}
$$

where $\mathbf{R}-\sigma^{2} \mathbf{I}_{M}=\mathbf{S}(\Theta) \Phi_{S} \mathbf{S}(\Theta)^{H}$ is the signal-part of the true covariance matrix and $(\cdot)^{\#}$ denotes the Moore-Penrose pseudoinverse. Based on this identity in [10], and noting that we can express

$$
\left(\mathbf{R}-\sigma^{2} \mathbf{I}_{M}\right)^{\#}=\mathbf{E}_{S}\left(\boldsymbol{\Lambda}_{S}-\sigma^{2} \mathbf{I}_{K}\right)^{-1} \mathbf{E}_{S}^{H}
$$

the authors proposed to use the following estimator for the signal power of the $k$ th source

$$
\hat{P}_{k}=\frac{1}{\mathbf{s}^{H}\left(\theta_{k}\right) \hat{\mathbf{E}}_{S}\left(\hat{\mathbf{\Lambda}}_{S}-\hat{\sigma}^{2} \mathbf{I}_{K}\right)^{-1} \hat{\mathbf{E}}_{S}^{H} \mathbf{s}\left(\theta_{k}\right)}
$$

where $\hat{\mathbf{\Lambda}}_{S}$ and $\hat{\mathbf{E}}_{S}$ contain the signal eigenvalues and eigenvectors of the sample covariance matrix respectively, and

$$
\hat{\sigma}^{2}=\frac{1}{M-K} \sum_{k=1}^{M-K} \hat{\lambda}_{k}
$$


is an estimator of the noise power. Interestingly enough, this estimator only depends on the signature of the source of interest $\mathbf{s}\left(\theta_{k}\right)$ and consequently it is robust to the presence of outliers in the estimation of the DoAs of the other sources. Furthermore, its implementation is relatively simple if the eigendecomposition of $\hat{\mathbf{R}}$ has been performed in a previous stage. Indeed, note that we can express

$$
\hat{\mathbf{E}}_{S}\left(\hat{\boldsymbol{\Lambda}}_{S}-\hat{\sigma}^{2} \mathbf{I}_{K}\right)^{-1} \hat{\mathbf{E}}_{S}^{H}=\sum_{m=M-K+1}^{M} \frac{1}{\hat{\lambda}_{m}-\hat{\sigma}^{2}} \hat{\mathbf{e}}_{m} \hat{\mathbf{e}}_{m}^{H}
$$

and therefore

$$
\hat{P}_{k}=\frac{1}{\sum_{m=M-K+1}^{M} \frac{1}{\hat{\lambda}_{m}-\hat{\sigma}^{2}}\left|\mathbf{s}^{H}\left(\theta_{k}\right) \hat{\mathbf{e}}_{m}\right|^{2}}
$$

which can be computed very efficiently without matrix inversions.

However, the main problem with this estimator is the fact that $\mathbf{s}^{H}\left(\theta_{k}\right) \hat{\mathbf{E}}_{S}\left(\hat{\mathbf{\Lambda}}_{S}-\hat{\sigma}^{2} \mathbf{I}_{K}\right)^{-1} \hat{\mathbf{E}}_{S}^{H} \mathbf{s}\left(\theta_{k}\right)$ is a consistent estimator of $\mathbf{s}^{H}\left(\theta_{k}\right)\left(\mathbf{R}-\sigma^{2} \mathbf{I}_{M}\right){ }^{\#} \mathbf{s}\left(\theta_{k}\right)$ only when $N \rightarrow \infty$ for a fixed $M$. As a consequence of this, it turns out that the performance is quite poor whenever $M, N$ have the same order of magnitude (cf. Section 5) and that a much better estimation can be obtained using (5). In the next section, we present a new estimator that holds the advantageous properties of $\hat{P}_{k}$ (namely, it is easily implemented without matrix inversions and it is robust to the presence of outliers), but it has a better performance than $\hat{P}_{k}$ in finite sample size situations. This is because the estimator is designed to be consistent when both $M, N$ are large but have the same order of magnitude. We will see in Section 5 that this guarantees a good performance in the finite sample size regime, comparable to the GML estimator in (5), with the added features of simplicity and robustness against outliers in the DoA detection process.

\section{AN $M, N$-CONSISTENT POWER ESTIMATOR}

In order to derive a power estimator that is consistent as $M, N$ grow without bound at the same rate, we need the following assumptions:

(As1) The observation vectors $\mathbf{y}(n)$ can be modeled as $\mathbf{y}(n)=\mathbf{R}^{1 / 2} \mathbf{u}(n)$, where $\mathbf{R}^{1 / 2}$ is a positive definite Hermitian square-root of $\mathbf{R}$, and $\mathbf{u}(n), n=1 \ldots N$, is a collection of independent and identically distributed complex $M \times 1$ random vectors. The real and imaginary parts of the entries of $\mathbf{u}(n)$ are all i.i.d. absolutely continuous random variables with zero mean, variance $1 / 2$ and finite $8+\epsilon, \epsilon>0$, moments.

(As2) The $M \times 1$ steering vector s $(\theta)$ has uniformly bounded norm for all $M$, that is

$$
\sup _{M} \sup _{\theta}\|\mathbf{s}(\theta)\|<+\infty
$$

where $\|\cdot\|$ here denotes Euclidean norm.

(As3) The asymptotic number of snapshots per sensor/antenna $(1 / c)$ is higher than the parameter $\xi$ defined as

$$
\xi \equiv \frac{1}{M} \sum_{m=1}^{M}\left(\frac{\lambda_{m}}{\lambda_{m}-\bar{f}}\right)^{2}
$$

where $\bar{f}$ denotes the smallest real-valued solution to the equation

$$
\frac{1}{M} \sum_{m=1}^{M} \frac{\lambda_{m}^{2}}{\left(\lambda_{m}-f\right)^{3}}=0
$$

Theorem 1 Under (As1 - As3), the following estimator of the power of the kth source is strongly consistent as $M, N \rightarrow \infty$ at the same rate,

$$
\check{P}_{k}=\frac{1}{\mathbf{s}^{H}\left(\theta_{k}\right)\left(\sum_{m=1}^{M} \psi(m) \hat{\mathbf{e}}_{m} \hat{\mathbf{e}}_{m}^{H}\right) \mathbf{s}\left(\theta_{k}\right)}
$$

where $\psi(m), m=1 \ldots M$ are the coefficients ${ }^{1}$

$\psi(m)= \begin{cases}-\frac{1}{\check{\sigma}^{2}} \sum_{k=M-K+1}^{M}\left(\frac{\hat{\vartheta}_{k}}{\hat{\lambda}_{m}-\hat{\vartheta}_{k}}-\frac{\hat{\lambda}_{k}}{\hat{\lambda}_{m}-\hat{\lambda}_{k}}\right) & m \leq M-K \\ \frac{1}{\check{\sigma}^{2}}\left(\sum_{k=0}^{M-K} \frac{\hat{\vartheta}_{k}}{\hat{\lambda}_{m}-\hat{\vartheta}_{k}}-\sum_{k=1}^{M-K} \frac{\hat{\lambda}_{k}}{\hat{\lambda}_{m}-\hat{\lambda}_{k}}\right) & m>M-K .\end{cases}$

Here $\check{\sigma}^{2}$ is an estimator of the noise power $\sigma^{2}$ that is also strongly consistent as $M, N \rightarrow \infty$ at the same rate

$$
\check{\sigma}^{2}=\frac{N}{M-K} \sum_{k=1}^{M-K}\left(\hat{\lambda}_{k}-\hat{\mu}_{k}\right),
$$

$\hat{\mu}_{1} \leq \hat{\mu}_{2} \leq \ldots \leq \hat{\mu}_{M}$ are defined in Section 2, and $\hat{\vartheta}_{0} \leq \hat{\vartheta}_{1} \leq$ $\ldots \leq \hat{\vartheta}_{M}$ are the real-valued solutions to the following equation in $\hat{\vartheta}$

$$
\hat{\vartheta}=\check{\sigma}^{2}\left(1-c \frac{1}{M} \sum_{k=1}^{M} \frac{\hat{\lambda}_{k}}{\hat{\lambda}_{k}-\hat{\vartheta}}\right) .
$$

When $c>1$, we use the convention $\hat{\vartheta}_{0}=\ldots=\hat{\vartheta}_{[M-N]^{+}}=$ 0 whereas $\hat{\vartheta}_{[M-N]^{+}+1}, \ldots, \hat{\vartheta}_{M}$ are the positive solutions to the above equation.

Proof. The proof follows directly from the derivations in [11].

\section{NUMERICAL EVALUATION}

We consider a scenario with two sources impinging on a uniform linear array of $M=20$ elements separated half a wavelength from DoAs of 35 and 37 degrees with a power $15 \mathrm{~dB}$ and $10 \mathrm{~dB}$ above the noise floor respectively. The number of available samples was $N=35$, and the power estimation was performed after applying the GMUSIC estimation technique for the two DoAs. Figures 1 and 2 represent the histogram obtained from $10^{5}$ realizations of the three power estimators considered herein. In particular, Figure 1 shows the histogram corresponding to the realizations where the two sources were correctly detected, whereas Figure 2 represents the same quantity for the realizations where only the signal of interest (that is, the one for which the power is estimated) was correctly detected while the other DoA estimation was estimated as an outlier. In this simulation, an outlier was declared whenever the detected and the true DoAs were separated more than 0.5 degrees. Apart from the performance of the proposed power estimator in (9) (solid line) we also represent the performance of the ML estimator in (5) (dotted line) and the traditional form of our estimator as given in (6) (dashed line).

Observe that, when there are no outliers in the DoA detection process, our estimator presents a behavior very close to the ML power estimator, and much better than the traditional form of the estimator in (6). Furthermore, when only the DoA of the signal of interest is correctly detected while the other one is declared as an outlier, our estimator is able to provide much better estimates

\footnotetext{
${ }^{1}$ In the expression of $\psi(m)$ we use the convention that any term of the form $0 / 0$ is identically zero.
} 
than the other two (see further the lower plot in Figure 2). Again, this is thanks to the fact that the proposed power estimator only depends on the signature of the source of interest. Therefore, an outlier in the DoA detection of the other source does not affect its performance.

\section{CONCLUSIONS}

We have presented a new source power estimator that is suitable for scenarios with low sample size constraints. The estimator is constructed from the eigenvalues and eigenvectors of the sample covariance matrix, and it is therefore especially indicated when the DoA detection process is carried out using a subspace based technique. Because the estimator only depends on the spatial signature of the user of interest, it is robust to the presence of outliers in the DoA detection process of the other sources. Simulations demonstrate that the performance in the absence of outliers is very similar to that of the ML technique, with significantly lower computational complexity (once the eigendecomposition of the sample covariance matrix is available).
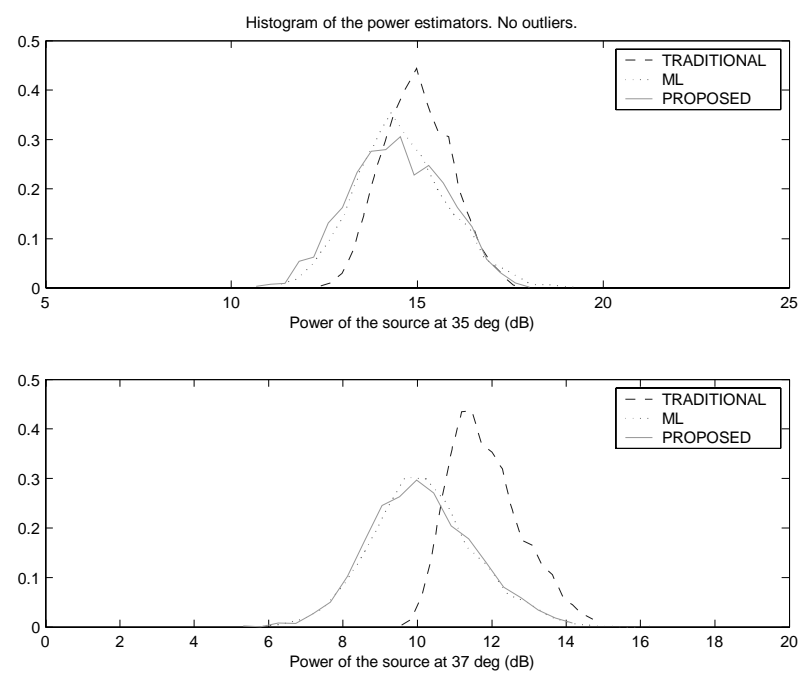

Fig. 1. Histogram of the realizations of the power estimators when there are no outliers in the DoA detection stage.

\section{REFERENCES}

[1] D. Tufts, A. Kot, and R. Vaccaro, "The threshold analysis of SVD-based algorithms," in Proceedings of the IEEE International Conference on Acoustics, Speech and Signal Processing, pp. 2416-2419, 1988. (Vol. 4).

[2] D. Tufts, A. Kot, and R. Vaccaro, The Threshold Effect in Signal Processing Algorithms Which Use an Estimated Subspace, in SVD and Signal Processing, II: Algorithms, Analysis and Applications. New York: Elsevier, 1991.

[3] R. Schmidt, "Multiple emitter localization and signal parameter estimation," in Proceedings of the RADC, Spectral Estimation Workshop,, (Rome (NY)), pp. 243-258, 1979. Reprinted in "Modern Spectral Analysis II", S.B. Kesler (ed.), IEEE Press, New York, 1986.
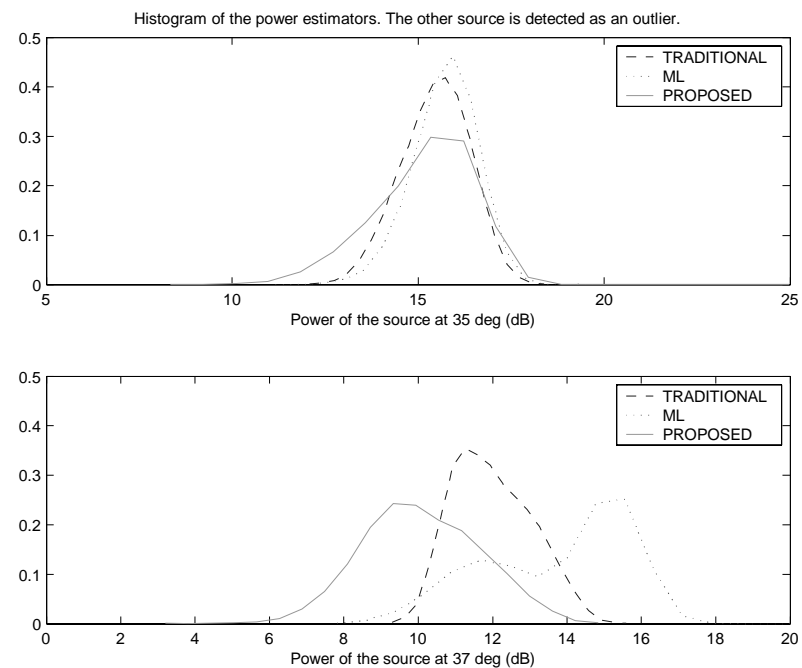

Fig. 2. Histogram of the realizations of the power estimators when the DoA of the additional source is detected as an outlier.

[4] G. Bienvenu, "Influence of spatial coherence of the background noise on high resolution passive methods," in Proceedings of the IEEE International Conference on Acoustics, Speech and Signal Processing, (Washington DC), pp. 306309, 1979.

[5] Y. Abramovich, D. Gray, A. Gorokhov, and N. Spencer, "Positive-definite Toeplitz completion in DOA estimation for nonuniform linear antenna arrays- Part I: Fully augmentable arrays," IEEE Transactions on Signal Processing, vol. 46, pp. 2458-2471, 1998.

[6] X. Mestre and F. Rubio, "An improved subspace-based algorithm in the small sample size regime," in Proceedings of the IEEE International Conference on Acoustics, Speech and Signal Processing, (Toulouse (France)), 2006.

[7] X. Mestre, "An improved weighted MUSIC algorithm for small sample size scenarios," in Proc. Adaptive Sensor Array Processing Workshop, (Boston (MA)), 2006.

[8] V. Girko, An Introduction to Statistical Analysis of Random Arrays. The Netherlands: VSP, 1998.

[9] B. Ottersten, M. Viberg, P. Stoica, and A. Nehorai, Exact and Large Sample ML Techniques for Parameter Estimation and Detection in Array Processing, ch. 4, pp. 99-151. Radar Array Processing, Berlin: Springer-Verlag, S. haykin and L. litva and T. shepherd ed., 1993.

[10] M. McCloud and L. Scharf, "A new subspace identification algorithm for high resolution DOA estimation," IEEE Transactions on Antennas and Propagation, vol. 50, pp. 13821390, Oct. 2002.

[11] X. Mestre and M. Lagunas, "Modified subspace algorithms for DoA detection in the small sample size regime," To be submitted, 2006. Temporarily available at http://www.cttc.cat/drafts/cttc-rc-2006-003.pdf. 